\title{
The outcomes of a school-based intervention for depressive symptoms in adolescents do not echo the promising findings of earlier studies
}

\author{
Sally Merry \\ Department of Psychological Medicine, University of Auckland, Auckland, New Zealand
}

\section{WHAT IS ALREADY KNOWN ON THIS TOPIC?}

Depressive disorder, which frequently starts in adolescence, is costly both to individuals and to society at large. ${ }^{1}$ Most depression in adolescents is untreated. ${ }^{2}$ A number of studies have investigated classroombased prevention and early intervention programmes to address the problem. ${ }^{3}$ While results from a number of randomised controlled trials are promising, most studies do not include an active comparison group and there has been little research demonstrating that these interventions remain effective when moved from research settings into the real world.

\section{WHAT THIS PAPER ADDS?}

- In this study, the intervention, which has some evidence of efficacy, was compared with the usual class curriculum and with an attention control group in a classroom setting, using a model of delivery that could be scaled up for national dissemination. Care was taken to address some of the weaknesses of previous studies with rigorous trial design conducted in a real-world setting, testing a model that could be implemented in practice and, most particularly, the use of an attention control group.

- Disappointingly, the positive results from the earlier trials ${ }^{4}{ }^{5}$ were not replicated. Few previous studies used an attention control group and many preventive interventions have been studied in conditions that would be difficult to replicate.

- A potential for harm was identified, with symptoms reported at 12 months following the intervention significantly higher than after attendance at usual classes. This is a new and sobering finding.

\section{LIMITATIONS}

- Only a short, albeit validated, self-report scale (Short Mood and Feelings Questionnaire) was used to assess depressive symptoms, meaning that assessment of the primary outcome measure was not blind.
- No measure was taken of depressive episodes over the follow-up period. A cross-sectional report of symptoms by young people may miss real difference in period prevalence of depressive episodes.

\section{WHAT NEXT IN RESEARCH?}

Future studies should include the use of an attention control group and an estimate of period prevalence of depressive episodes following intervention. The potential for harm should be considered and measured. Given the modest results to date, the search for more effective interventions or modes of delivery should continue.

\section{DO THESE RESULTS CHANGE YOUR PRACTICES AND WHY?}

No. This is a study that is primarily of interest to those implementing public health interventions. These results suggest that it would be premature to roll out depression prevention or early intervention programmes in classroom settings.

\section{Competing interests None.}

doi:10.1136/eb-2014-101718

\section{REFERENCES}

1. Ferrari AJ, Charlson FJ, Norman RE, et al. Burden of depressive disorders by country, sex, age, and year: findings from the global burden of disease study 2010. PLoS Med 2013;10:e1001547.

2. Kataoka SH, Zhang L, Wells KB. Unmet need for mental health care among US children: variation by ethnicity and insurance status. Am J Psychiatry 2002;159:1548-55.

3. Merry SN, Hetrick SE, Cox GR, et al. Psychological and educational interventions for preventing depression in children and adolescents. Cochrane Database Syst Rev 2011; 12:CD003380

4. Merry S, Mcdowell H, Wild C, et al. A randomized placebo-controlled trial of a school-based prevention program. J Am Acad Child Adolesc Psychiatry 2004; 43:538-47

5. Shochet I, Dadds M, Holland D, et al. The efficacy of a universal school-based program to prevent adolescent depression. J Clin Child Psychol 2001;30:303-15.

ABSTRACT FROM: Stallard P, Phillips R, Montgomery AA, et al. A cluster randomised controlled trial to determine the clinical effectiveness and cost-effectiveness of classroom-based cognitive-behavioural therapy (CBT) in reducing symptoms of depression in high-risk adolescents. Health Technol Assess 2013;17:vii-xvii, 1-109.

Participants In total, 1064 adolescents aged 12-16 years, attending personal, social and health education (PSHE) classes who were classified as being at high risk for depression (persistent score $\geq 5$ on the Short Mood and Feelings Questionnaire (SMFQ)).

Setting Eight state secondary schools in the UK.

Intervention Classroom-based cognitive behavioural therapy (CBT; $n=392)$ versus usual PSHE curriculum $(n=298)$ versus an attention control PSHE group $(n=374)$. The CBT intervention used the Resourceful Adolescent Programme (RAP), adapted for UK use. The RAP is a manualised depression prevention programme designed for use with groups of young people that identifies and challenges negative and dysfunctional thoughts and social problems, and promotes awareness of personal strengths and problem-solving. CBT was delivered by two trained external facilitators to the whole class at weekly or fortnightly intervals for an average of 9 weeks, though this was adaptable to fit in with each school's curriculum.

Comparison The usual school PSHE curriculum was provided by school teachers with no input from the research team. PSHE is a programme of learning aimed at helping schoolchildren acquire the knowledge, understanding and skills they need to manage their lives. Attention control PSHE was delivered by teachers assisted by two trained external facilitators. It involved similar time and contact with facilitators as the CBT group, but without CBT components.

Patient follow-up $76 \%$ of the CBT group, $82 \%$ of the attention control PSHE group and $81 \%$ of the usual PSHE group.

Allocation Randomisation was by year group, stratified by number of classes, students and PSHE scheduling; every school had at least 1 year group allocated to CBT. 
Blinding Unblinded.

\section{OUTCOMES}

Symptoms of depression at 12 months (SMFQ)

Across all arms, there was an overall decrease in symptoms of depression between baseline and 12 months $(p<0.001)$. There was no significant difference between intervention arms (CBT vs usual PSHE adjusted difference in means $0.97,95 \%$ CI -0.34 to 2.28; CBT vs attention control PSHE $-0.63,95 \%$ CI -1.99 to $0.73)$.

Thoughts about personal failure (Children's Automatic Thoughts Scale)

There was suggestion that CBT could have a potentially harmful effect compared with usual PSHE for thoughts about personal failure at 12 months, but the effect was non-significant $(\mathrm{OR}=1.95$, $95 \%$ CI 0.25 to 3.66 ). 